\title{
Les discours de réception du marquis de Marnésia à l'académie de Besançon : un art de l'écart
}

Julie LABLANCHE

Docteur en Langue et littérature françaises, UBFC

\begin{abstract}
I77o, le marquis de Lezay-Marnésia, de vieille noblesse comtoise, est admis à l'académie de Besançon fondée vingt-cinq ans plus tôt. Membre associé, puis titulaire du onzième fauteuil, il adresse à ses confrères des discours de réception dans lesquels le respect des normes génériques se caractérise par son instabilité. Le marquis sait à l'évidence aussi bien appliquer les codes que s'en affranchir. Commettant, au sein d'un cadre formel contraint, des écarts qui vont de l'entorse sans gravité à la transgression impertinente, il offre avec ses deux discours l'exemple d'une voix singulière, dont on peut faire l'hypothèse qu'elle surprit probablement une majorité de ses confrères bisontins.
\end{abstract}

Keywords: Lezay-Marnésia, académie de Besançon, discours de réception, épidictique.

La fondation d'une académie des sciences, belles-lettres et arts à Besançon en 1752 permet à la province comtoise de rejoindre un réseau de sociétés savantes déjà solidement implanté en France à cette époque. Placée sous la protection du duc de Tallard, gouverneur de Franche-Comté, qui avait intercédé en faveur de sa création ${ }^{\mathrm{I}}$, la nouvelle académie compte à ses débuts quarante membres titulaires puis, très vite, s'élargit à des classes d'associés$^{2}$. Elle se lance immédiatement dans des travaux variés et aiguillonne l'émulation de ses concitoyens : les cinquante-trois volumes in-folio qu'elle constitue jusqu'en 1789 (dont trente-sept réunissent les copies ayant concouru pour les prix annuels) sont le reflet de cette activité et de ce rayonnement ${ }^{3}$. Notre travail de doctorat ${ }^{4}$ a pu faire émerger la part littéraire de cet immense corpus, resté partiellement inédit, en se centrant sur la pratique de l'éloquence institutionnelle, et plus spécifiquement épidictique, au sein de cette académie comtoise. Dans cette perspective, les discours de récep-

I Les ducs de Duras seront, à sa suite, gouverneurs et protecteurs de l'académie.

2 Dès I757, l'académie se choisit douze associés comtois et étrangers. Leur nombre est encore doublé à la fin du siècle.

3 Ce fonds est conservé à la Bibliothèque municipale d'étude et de conservation de Besançon. Il est décrit et consultable sous forme numérisée sur le site Mémoire vive (http://www. memoirevive.besancon.fr $>$ ). Voir en particulier ms.academie.3 (discours de réception de Marnésia, I777) et ms.academie.8 (discours de réception de Marnésia, I778).

4 Éloges inédits de l'académie des sciences, belles-lettres et arts de Besançon (I752-I789). Soutenue le 7 novembre 2017 sous la direction de France Marchal-Ninosque. 
tion, que les archives conservent en nombre important ${ }^{5}$, fournissent un matériau précieux pour l'étude. Officialisant en quelque sorte la naissance d'un académicien, ce type de discours se caractérise en effet par une teneur laudative si marquée qu'on a pu parler à son sujet de « cascade élogieuse $»^{6}$. Nous avons transcrit et annoté plus d'une quarantaine de ces discours qui étaient copiés in extenso dans les archives comtoises et n'avaient pas encore été publiés. Or, de cette série épidictique, il nous semble que deux pièces en particulier méritent d'être signalées pour leur propension à osciller entre le respect des contraintes génériques et une certaine forme d'indépendance à l'égard de ces mêmes contraintes : il s'agit des discours du marquis de Marnésia, reçu à la fin de la décennie I770, personnage dont il faut retracer brièvement la trajectoire académique ${ }^{7}$.

Claude-François-Adrien, marquis de Lezay-Marnésia, naît à Metz le 24 août 1735 d'un père de vieille noblesse comtoise, François-Gabriel de Lezay-Marnésia, brigadier des armées du Roi, et d'une mère lettrée, Charlotte-Antoinette de Bressey, familière de la cour de Lorraine et salonnière ${ }^{8}$. La tradition familiale conduit le jeune marquis de Marnésia à embrasser la carrière militaire tout en cultivant la littérature9. Lié à Saint-Lambert, Cerutti, Fontanes, Boufflers et Chamfort, il est successivement adopté par les académies de Nancy (I767) et de Lyon (1774) avant d'être distingué par celle de Besançon, dont faisait partie depuis I772 l'évêque d'Évreux, son oncle $^{\mathrm{IO}}$. Ayant démissionné de l'armée, le marquis s'installe en I769 dans ses terres jurassiennes. L'académie le choisit d'abord pour occuper une place d'associé comtois ${ }^{\text {II }}$ puis le nomme titulaire quelques mois plus tard ${ }^{12}$, en remplacement du défunt marquis de Calviers ${ }^{13}$. Marnésia se rend dans la capitale comtoise à ces deux occasions, pour prononcer un discours de réception au palais Granvelle, siège de l'académie. Par la suite, il s'illustre comme un membre actif de la compagnie, lui fait lecture de plusieurs pièces de sa composition, tant littéraires que scientifiques ; la préside en I779, honneur qui lui vaut de recevoir le marquis de Ségur ${ }^{14}$, l'abbé de Clermont-Ton-

5 La plupart du temps accompagnés d'une réponse.

6 Daniel Roche, Le Siècle des Lumières en province : académies et académiciens provinciaux: 1680I789, Paris, EHESS, 1989 [1978], p. I70.

7 Nous nous appuyons en partie sur l'ouvrage de Roland Guy Bonnel, Éthique et esthétique du retour à la campagne au XVIII siècle : l'ouvre littéraire et utopique de Lezay-Marnésia, I735-I80o, New-York, Peter Lang, 1995, pp. 90-92.

8 Auteur de Lettres de Julie à Ovide, roman épistolaire paru anonymement en 1753.

9 Il s'illustre dès sa jeunesse en composant un article pour l'Encyclopédie, un éloge de Sully pour l'Académie française, un conte moral intitulé L'Heureuse Famille.

Io Claude-Louis-Albert de Lezay-Marnésia (I707-I790), évêque d'Évreux de I759 à I773.

II Le I2 mars I777.

I2 Le 7 janvier 1778 .

I3 Charles-François de Calviers (I693-I777), membre fondateur de l'académie comtoise.

I4 Philippe-Henri de Ségur (I724-I80I), reçu le 29 juin I779. La réponse du marquis de Mar- 
nerre $^{15}$ et le vicomte de Toulongeon ${ }^{16}$; continue de participer aux travaux collectifs après avoir quitté la présidence, intégrant en $1780^{17}$ le bureau chargé d'examiner les ouvrages relatifs à l'agriculture (domaine qui lui est cher), au commerce et à l'industrie, d'une part, et le bureau d'éloquence, de poésie et des beaux-arts, d'autre part ; réforme enfin son habitude de passer hors de Franche-Comté la mauvaise saison et reste à Besançon plusieurs hivers de suite. Des « affaires » dont la nature n'a pas été élucidée ${ }^{18}$ le rappellent cependant bientôt à Paris : cette évolution de sa situation personnelle le pousse à demander, au printemps $\mathrm{I} 78 \mathrm{I}$, à passer dans la classe des honoraires ${ }^{19}$. Sa demande exaucée le 12 décembre $\mathrm{I78}$ I, le marquis profite néanmoins dès l'hiver suivant des droits attachés à son nouveau statut : revenant visiter ses confrères bisontins, il leur lit un poème ${ }^{20}$, et assiste encore à plusieurs séances jusqu'en I784, où il paraît pour la dernière fois au palais Granvelle. Ces sept années d'un engagement actif paraissent en soi suffire à marquer les mémoires. Cependant, sans même considérer l'ensemble des travaux académiques du marquis, on peut raisonnablement avancer que ses discours de réception ont à eux seuls fait date à l'académie comtoise, le second plus encore que le premier, car le marquis s'y affranchit par moment des contraintes génériques. Nous nous proposons ici d'examiner plus en détail ces deux morceaux d'éloquence afin d'en montrer la percutante originalité, susceptible d'avoir dérouté les représentants de l'institution.

\section{Un éloge mis pour un autre}

Le discours que prononce Marnésia pour son association, le $\mathrm{I}^{\mathrm{er}}$ mai $\mathrm{I} 777$, est le plus court. Il débute de façon très conventionnelle. Après avoir célé-

nésia est imprimée dans la Séance publique extraordinaire de l'académie des sciences, belles-lettres et arts de Besançon, du 29 juin 1779.

I5 Anne-Antoine-Jules de Clermont-Tonnerre (I749-I830), reçu à la même séance. La réponse est également imprimée.

I6 François-Emmanuel de Toulongeon (I748-I8I2), reçu le 6 décembre I779. Son discours de réception et la réponse du marquis de Marnésia sont reproduits dans notre thèse de doctorat (publication en cours).

I7 Séances du 26 janvier et du 23 février 1780.

I8 Financières ou matrimoniales. R. Bonnel explique qu'en l'absence de sources, on ne peut pas trancher (op. cit., p. 9I).

I9 Les académiciens titulaires sont soumis au devoir de résidence en vertu d'une lettre de cachet du roi en date du 5 février I757. L'ancien évêque d'Évreux est lui aussi nommé honoraire en 1779 car, n'ayant pas établi sa résidence à Besançon, il se trouve trop éloigné de l'académie pour contribuer à ses travaux.

20 Séance du 6 décembre 1782 , Il s'agit d'un « Poème sur les jardins » imprimé sous le titre «Les Paysages » dans l'Essai sur la nature champêtre, Paris, Prault, 1787. Jacques Delille (académicien comtois lui aussi) s'était servi dès 1782 du titre Les Jardins (Les Jardins ou l'Art d'embellir les paysages, poème, Paris, Valade, $\mathrm{I} 782$ ). 
bré les sociétés littéraires, envisagées comme espaces de communion des savants et comme organes de communication des savoirs, le marquis s'interroge sur les droits qui l'autorisent à « oser pénétrer dans ce sanctuaire ». L'humble réponse qu'il avance aussitôt est parfaitement conforme à ce qui est attendu d'un récipiendaire : ces droits, avoue-t-il, ce sont « les bontés » de ses nouveaux confrères : « je n'en connais point d'autres; et pour les justifier, je n'ai que ma reconnaissance». Il est habituel, dans un discours de réception, de minorer ainsi ses propres talents et de soutenir que seule la bienveillance, «la grâce », «l'indulgence » ou la " prévention trop libérale » des autres académiciens ont pu déterminer l'adoption : c'est ce que prétendent bien d'autres Comtois fraîchement élus ${ }^{21}$. À cette ouverture répond une clôture tout aussi topique. Pour s'être déclaré dépourvu de talents, le récipiendaire ne doit pas moins justifier son élection et conforter ainsi le choix de l'académie. Les protestations d'humilité débouchent donc sur la formulation d'une promesse : celle de fournir à l'académie érigée en modèle des preuves rapides et tangibles de son zèle et de sa gratitude. Marnésia déclare ainsi : " guidé par vos leçons, et surtout enflammé par la reconnaissance, j'oserai, quoique d'un pas timide et mal assuré, vous suivre dans vos travaux, et peut-être qu'un jour je ne rougirai plus de n'avoir que des fleurs champêtres à vous présenter »- «fleurs " poétiques en l'occurrence, le marquis annonçant le premier chant de son « Poème sur les jardins ». La pudeur affichée ${ }^{22}$ est bien dans la veine d'un discours d'intronisation qui introduit avec toute la modestie requise le premier tribut du nouvel académicien.

Entre les deux seuils prend place un passage beaucoup plus original qui représente, quantitativement, près de la moitié du discours. Marnésia y évoque le plaisir qu'il éprouve d'être reçu lors d'une séance publique à laquelle assistent les officiers du régiment du Roi :

C'est sous les yeux des compagnons, des amis de ma jeunesse, que vous récompensez ce goût pour les arts, cet amour pour les lettres, que leurs talents et leur exemple ont allumé dans moi. Je les ai vus - le souvenir m'en est bien cher - autour d'un chef qui savait à la fois être leur guide et leur père, qui donnait à l'obéissance la rapidité du zèle parce qu'il semblait ne montrer que le désir de l'amitié, qui gravait dans les cœurs l'amour et le respect du devoir parce qu'il ne faisait jamais parler que l'honneur et son exemple - qui forçaient à chérir son autorité -, parce qu'il ne craignait pas de la compromettre en unissant les grâces à la raison et le sentiment à la fermeté. Je les ai vus dans le tumulte des camps, parmi les fatigues de la guerre, trouver

2I Tels le conseiller Guillemin de Vaivre et le président Terrier (en I765), le comte de Roussillon (en I768), l'abbé Guillemin (en I774), le président Mareschal de Vezet (en I778).

22 On a vu plus haut que Marnésia propose ultérieurement à ses confrères d'écouter d'autres extraits de ce fameux " Poème sur les jardins » (lors des séances du 6 décembre 1779 et du 6 décembre 1782 ). 
leurs délassements dans les arts de la paix ; faire même succéder aux plus pénibles travaux l'étude des sciences les plus profondes, et suivre en même temps les traces de Balon et celles de Vauban. Si j'avais su mieux profiter à leur école, Messieurs, j'aurais pu devenir digne de vous ; mais du moins ils ont formé mon âme à la sensibilité et m'ont appris à aimer.

L'intérêt du passage est bien sûr d'ordre biographique ${ }^{23}$, mais tient aussi à d'autres raisons. Rares sont dans les discours d'installation les allusions faites à l'assistance ; celles qu'on relève sous la plume d'autres impétrants sont plus fugitives que celle du discours qui nous occupe ${ }^{24}$. À l'évidence, l'allusion très appuyée de Marnésia à ses anciens compagnons d'armes prouve que l'éloquence d'apparat sait "répond[re] aux exigences de l'occasion qui [la] fait naître $\aleph^{25}$ : la présence des officiers du Roi à la séance publique, désignée comme un événement exceptionnel, justifie la particularité du discours ${ }^{26}$. Mais cette adaptation aux circonstances a pour corollaire une moindre observation des usages en vigueur. Alors qu' il convient en principe à un récipiendaire de célébrer l'institution qui l'accueille, cette célébration reste chez Marnésia en deçà de ce qu'elle est dans la plupart des discours du corpus. Il est notamment intéressant de constater que le marquis prête aux officiers du Roi une influence décisive sur sa propre formation, et même une capacité assez inattendue, de la part de personnalités extra-académiques, à le rendre "digne » des académiciens comtois. Certes, valeurs militaires et nobiliaires se croisent et certainement trouvent un écho auprès de la compagnie comtoise, dont le recrutement est largement aristocratique ; mais on s'attendrait à ce que soit davantage souligné le rôle d'aiguillon joué par l'académie elle-même - dans sa dimension savante - dans le parcours littéraire et scientifique du marquis. Celui-ci, en attribuant plus de mérite en la matière à ses amis de jeunesse qu'à ses nouveaux confrères, s'écarte décidément de la voie commune. L'originalité de Marnésia ne s'arrête pas là : elle bascule dans la transgression, car l'hommage de circonstance aux officiers se substitue en fait à un autre éloge : celui du duc de Tallard. Cet éloge, ritualisé à Besançon, est explicitement exigé par le règlement de la compagnie $^{27}$. Pourtant, jamais Marnésia ne cite le fondateur de l'académie; le seul «chef » révéré dans son discours est le comte de Guerchy. Cette omission, et

23 R. Bonnel souligne qu'il «permet de mesurer la dette qu'il [Marnésia] reconnait envers la tradition militaire» (op. cit., p. I4).

24 L'abbé Jacques se réjouit d'être reçu en présence de « l'assemblée la plus respectable (en I769), l'abbé Guillemin salue « un concours [...] brillant et [...] nombreux » (en I774).

25 Pierre Zoberman, Les Cérémonies de la parole. L'éloquence d'apparat en France dans le dernier quart du XVII siècle, Paris, Champion, I998, p. 20.

26 Du reste, Droz, qui reçoit Marnésia, salue dès son discours d'ouverture " cette cohorte également brave et brillante » dont la présence ajoute à l'éclat de la cérémonie.

27 Règlement pour la discipline intérieure de l'académie, article premier, Besançon, Daclin, I753, p. I. 
la primauté accordée à la situation d'énonciation sur le respect des statuts, confèrent à son discours une tonalité toute personnelle et non académique. Marnésia est alors l'un des premiers récipiendaires à faire l'économie des louanges dues à Tallard ${ }^{28}$. On ignore si cette infraction a été relevée, commentée ou critiquée par ses confrères, qui étaient prévenus en sa faveur ${ }^{29}$; il semble cependant qu'elle n'ait guère porté préjudice au marquis, puisqu'il est élu à l'unanimité (par onze votants) membre titulaire quelques mois plus tard.

\section{Le genre questionné}

Son changement de statut l'amène à prononcer un second discours d'installation devant la compagnie comtoise. Marnésia confirme alors sa capacité à faire se côtoyer dans un discours de réception les réflexions les plus conventionnelles et les écarts les plus inattendus. Comme celui de I777, le discours du 24 août 1778 s'ouvre en respectant les usages : le marquis remercie la compagnie de l'avoir promu au rang de titulaire et déclare, sur le mode de la prétérition, son incapacité à exprimer pleinement ses sentiments : «Messieurs, en resserrant les nœuds qui déjà m’unissaient à vous, vous acquérez de nouveaux droits à mon zèle et à ma reconnaissance. Je voudrais vous peindre avec toute leur énergie ces sentiments dont mon âme est pénétrée, mais je l'essaierais en vain [...] ». Le procédé est commun à de nombreux autres récipiendaires qui, pareillement, soulignent l'embarras qu'ils éprouvent à traduire leur émotion, et protestent de leur impuissance à répondre convenablement à l'honneur qui leur est fait ${ }^{30}$. Mais Marnésia ne s'en tient pas, pour sa part, à déplorer une faiblesse personnelle, un défaut d'éloquence individuelle. Il poursuit en mettant en lumière une autre dif-

28 Si l'on considère les discours complets et inédits, seuls les abbés Jacques (en I769 et I774), Guillemin (en I774) et Poulin (I774) s'en sont abstenus avant lui, sur une trentaine de récipiendaires. L'éloge de Tallard sera de moins en moins systématique dans les années 1780 .

29 À la séance du 4 mars I777, l'académie présente Marnésia comme " une personne agréable ». Le I2 mars, l'ayant élu à l'unanimité par huit voix exprimées, elle se réjouit auprès du duc de Duras de s'être agrégé « un gentilhomme de notre province, qui ne peut qu'à tous égards décorer notre société».

30 Voir ainsi les discours du mathématicien Yard («je sens mon insuffisance à vous remercier dignement, et j'en suis humilié. Que ne m'est il donc permis de recueillir et vous répéter tout ce qui vous a été dit d'éloquent par ceux de cette illustre compagnie que vous y avez reçus depuis son établissement ! , I76I) ; du président Terrier ("connaissant tout le prix du bienfait que je reçois en ce jour, je me consolerais de la médiocrité des talents que j'apporte parmi vous si, du moins, ils me mettaient en état de vous témoigner toute ma reconnaissance, et si je trouvais des expressions qui pussent répondre à mes sentiments », I765); de l'abbé Dagay («il est bien difficile de rendre dans toute sa force un sentiment dont le cœur est pénétré. Je voudrais pouvoir vous exprimer l'extrême reconnaissance dont le mien est rempli, mais je n'espère pas y réussir si vous ne suppléez vous-même à la faiblesse de mes expressions », 1782). 
ficulté, qui consiste à s'inscrire dans une histoire déjà largement écrite du compliment académique :

depuis plus d'un siècle la première académie du royaume, sans cesse occupée à trouver de nouvelles tournures pour rendre toujours la même idée, a fait devenir lieux communs les expressions de la plus vive sensibilité. Les remerciements répétés par chacun de ses membres ne nous laissent que la facilité peu glorieuse de nous traîner sur leurs traces dans un genre qu'ils ont épuisé.

Cette réflexion sur l'usure du genre est d'une audacieuse originalité. Elle jette en effet le discrédit sur la qualité des discours qui continuent à être prononcés dans les différentes sociétés savantes du royaume, aussi bien en province qu'à Paris. À l'Académie française, les élections se succèdent régulièrement : les plus récentes sont à cette date celles de Jean-François de La Harpe ${ }^{31}$ et de l'abbé Millot (un Comtois) ${ }^{32}$, tandis que celle de Jean-François Ducis ${ }^{33}$ se profile à la fin de l'année i778. Inspirée par le grand modèle parisien, la compagnie comtoise n'a guère de chances d'être épargnée par la fadeur du pstittacisme : une telle insinuation, peu valorisante, est bien hardie de la part d'un récipiendaire. Marnésia relaie ainsi une critique qui, pour n'être pas neuve - dès les années I730, Voltaire raillait férocement les discours de réception dans les Lettres philosophiques ${ }^{34}$ - n'avait jamais été ainsi exposée auparavant dans l'académie de Besançon, en pleine cérémonie d'installation. Poursuivant sa réflexion métadiscursive, le marquis en vient à prôner d'autres moyens que le compliment rituel de témoigner sa reconnaissance à l'académie :

3I Élu le I3 mai I776 à la place de Charles-Pierre Colardeau.

32 Élu le 4 décembre I777 à la place de Gresset. L'abbé Millot avait reçu deux fois le prix d'éloquence de l'académie de Besançon, en 1755 et I759, sur les sujets : «Si le seul amour du devoir peut produire d'aussi grandes actions que le désir de gloire » et « C'est une marque de grandeur d'âme, lorsque les honneurs rendent un homme meilleur ».

33 Élu le 28 décembre 1778 à la place de Voltaire.

34 «L'usage s'est insensiblement établi que tout académicien répéterait ces éloges à sa réception; ç’a été une espèce de loi d'ennuyer le public. Si on cherche ensuite pourquoi les plus grands génies qui sont entrés dans ce corps ont fait quelquefois les plus mauvaises harangues, la raison en est encore bien aisée ; c'est qu'ils ont voulu briller, c'est qu'ils ont voulu traiter nouvellement une matière toute usée : la nécessité de parler, l'embarras de n'avoir rien à dire et l'envie d'avoir de l'esprit sont trois choses capables de rendre ridicule même le plus grand homme; ne pouvant trouver des pensées nouvelles, ils ont cherché des tours nouveaux, et ont parlé sans penser, comme des gens qui mâcheraient à vide, et feraient semblant de manger en périssant d'inanition. Au lieu que c'est une loi dans l'Académie française de faire imprimer tous ces discours, par lesquels seuls elle est connue, ce devrait être une loi de ne les imprimer pas » (Amsterdam, Lucas, Au Livre d'or, I734, pp. I2I-I22). 
Que faire donc : l'abandonner, ce genre devenu stérile à force d'avoir produit, comme on abandonne une mine dont il n'y a plus de diamants à tirer ? Mais faut-il renoncer au plaisir si touchant, au devoir si doux et si sacré de manifester son cœur quand il est le plus délicieusement affecté ? Non, Messieurs, en sacrifiant l'éclat éblouissant et passager de l'éloquence des mots, il nous reste un moyen digne de vous, des lettres et de la patrie, de nous montrer reconnaissant : partager vos travaux, nous enflammer comme vous du désir d'être utile, nous éclairer de vos lumières, voilà comme nous pourrons en quelque sorte justifier votre choix et nous acquitter.

Moment paradoxal, où le discours récuse le discours et prône le zèle académique comme seule manière idoine de remercier l'assemblée. L'unique ressource qui reste au marquis pour rester cohérent serait donc de se taire... Mais, rattrapé par les conventions, son propos bifurque brutalement : «Cependant, il est une obligation dont rien ne peut nous dispenser. Le sentiment l'impose et la remplit. Il rend à la mémoire de votre auguste fondateur un hommage simple et pur ; pour le louer, pour le faire chérir, il retrace ses bienfaits ». Suivent quelques lignes consacrées à l'intérêt pris par le duc de Tallard à la province comtoise, intérêt culminant dans la fondation de l'académie. L'évocation du devoir imposé au récipiendaire rappelle la façon dont Voltaire, une fois admis à l'Académie, sacrifiait également au rite pourtant honni du compliment oratoire ${ }^{35}$. Pour Marnésia, qu'il renoue avec cette ligne consensuelle est d'autant plus piquant qu'on a en tête son discours de I777, qui gardait le silence sur Tallard. Le marquis cherche peut-être cette fois, en se conformant au règlement, à faire oublier que lui-même y a manqué au moment de son association. Le rappel, si ferme et si explicite, de l'« obligation » où il se trouve n'est peut-être pas dénué de connivence : Marnésia soulignerait par un clin d'oeil complice, en quelque sorte, le fait qu'il se mette en règle et répare une lacune antérieure. Le passage fait écho en tout cas à bon nombre d'autres compliments de réception qui, évoquant eux aussi « l'obligation imposée à tout nouvel académicien » de louer le duc ${ }^{36}$, rappellent avec plus ou moins de détails quelle fut son action, et assurent que « le nom de Tallard sera à jamais gravé dans [les] cœurs » ${ }^{37}$.

35 « Je sais [...] que le public, toujours avide de nouveautés, pense que tout est épuisé sur votre fondateur et sur vos protecteurs ; mais pourrais-je refuser le tribut que je dois, parce que ceux qui l'ont payé avant moi ne m'ont laissé rien de nouveau à vous dire ? Il en est de ces éloges qu'on répète comme de ces solennités qui sont toujours les mêmes, et qui réveillent la mémoire des événements chers à un peuple entier ; elles sont nécessaires » (Discours de réception, Les CEuvres complètes de Voltaire, 30 A, Oxford, Voltaire foundation, 2003, p. 33).

36 Droz (en I763).

37 Labbé Nonnotte (en I78I). 


\section{Les deux pôles épidictiques}

Mais plus intéressante est la suite du discours de i778. L'évocation de Tallard débouche sur celle, tout aussi attendue, du marquis de Calviers, dont le fauteuil échoit à Marnésia ${ }^{38}$. En retraçant à grands traits la vie du défunt, en célébrant ses qualités morales et son goût pour la littérature et les arts, Marnésia satisfait aux usages. La conclusion à laquelle il aboutit semble d'abord topique : " héritier de sa place et non de ses talents, je ne puis lui assurer qu'une apothéose obscure ". Rien là que de très ordinaire à première vue; les formules analogues fourmillent dans les discours d'autres académiciens comtois ${ }^{39}$. Cependant, contrairement à ce qui se passe ailleurs, la minimisation de son talent d'orateur ne sert pas seulement à Marnésia à faire ressortir, par contraste, celui du défunt ou celui de l'institution qui le reçoit, supposée le guider vers les cimes de l'éloquence. Plutôt que de vanter les dispositions des académiciens comtois, il réserve en effet son admiration à l'Académie française. Il imagine en effet les honneurs funèbres que celleci aurait pu accorder au marquis de Calviers, si elle l'avait compté dans ses rangs :

Moins modeste, il en aurait eu [une apothéose], sans doute, une plus digne. S'il se fût fait présenter par l'un de ces génies qui maîtrisent les suffrages, s'il eût employé les femmes qui les entrainent, les portes du temple de l'immortalité se seraient ouvertes pour lui ; la plume de l'éloquence aurait tracé son éloge avec cette mâle énergie, cette pureté de goût, cette harmonie soutenue, ces traits véhéments, cette sagacité sûre, et surtout avec cette impartialité respectable et vraiment philosophique qui caractérisent l'orateur de la plus célèbre académie.

Cet orateur, à qui serait échu l'éloge du défunt marquis de Calviers, ne peut être que d'Alembert, secrétaire perpétuel de l'Académie française depuis I772. Or il est pour le moins inattendu que le marquis de Marnésia détourne ainsi l'hommage dû à son prédécesseur en faveur d'une figure qui jouit d'un maigre crédit au sein de la compagnie comtoise. Il est rarissime en effet que les académiciens bisontins se réclament de d'Alembert ou même empruntent à ses écrits dans leurs discours institutionnels. Certes, Droz le cite nommément dans un éloge funèbre ${ }^{40}$, et on note quelques convergences

38 Marnésia n'était en revanche pas tenu de faire l'éloge de son prédécesseur (l'abbé Poulin) en 1777 puisque ce dernier, bien vivant, venait d'être nommé titulaire.

39 Le conseiller Guillemin de Vaivre, successeur du marquis Dumesnil, s'exclame par exemple : «Mais je m’aperçois, Messieurs, que j'entame son éloge, et comment pourrais-je l'entreprendre, moi qui lui succède et ne le remplace point? "

40 Éloge du prince de Bauffremont. Le défunt ayant eu pour professeur Dumarsais, Droz renvoie par deux fois à l'éloge de ce dernier par d'Alembert. 
de vues entre d'Alembert et les Comtois ${ }^{41}$. Mais on ne peut oublier d'un autre côté une pique du secrétaire perpétuel Courbouzon visant "deux philosophes beaux esprits, dont l'un est géomètre par état [d'Alembert], et l'autre misanthrope par goût [Rousseau] $\aleph^{42}$, ni les attaques répétées contre le parti éclairé en général : l'abbé Guillemin dénonçant « ce que notre siècle décore si ridiculement du nom de philosophe ${ }^{43}$, Droz réprouvant " ces prétendus philosophes qui se plaisent dans l'illusion de quelques sophismes contre les vérités les plus sublimes de notre dogme, ou contre le gouvernement $»^{44}$, l'abbé Dagay fustigeant « une philosophie également destructrice de l'ordre moral et civil » ${ }^{45}$, le président de Vezet condamnant « ce fantôme imposteur",

cette philosophie nouvelle qui, franchissant toutes les bornes, ne respectant rien, a attaqué de la même main et l'autel et le trône, qui a porté dans les esprits le doute et l'incertitude, et qui romprait tous les liens de la société en flétrissant le cœur, et anéantirait les États en détruisant les principes qui en font l'union et l'harmonie ${ }^{46}$.

Cette ligne est la plus couramment défendue à l'académie comtoise : il est passablement rare qu'y soient chantées les louanges d'un représentant des Lumières. Le marquis de Marnésia fait preuve de singularité quand, après avoir critiqué les insipides discours de réception en vigueur dans les sociétés savantes, il préfère proposer à l'admiration de ses nouveaux confrères les talents d'encomiaste de d'Alembert : les éloges funèbres que compose ce dernier pour l'Académie française, très libres de ton et d'esprit, débordent parfois du cadre strictement encomiastique pour faire des incursions dans le blâme, et rebutent probablement des provinciaux accoutumés à révérer le modèle fontenellien. Est-ce sous l'influence des «traits véhéments » de d'Alembert ? Le comble de la transgression est atteint quand Marnésia pointe ce qui selon lui manque à la compagnie qui l'accueille :

Parmi vous, Messieurs, il faut l'avouer, il serait difficile de lui trouver un semblable panégyriste. Vous n'avez pas l'art heureux d'unir à l'éloquen-

4I Au sujet du recrutement des académiciens, et de l'embarras des panégyristes chargés parfois de faire l'éloge de personnalités « plus qu'éclatante[s] qu'indispensable[s] » (d'Alembert, Éloge de Jules de Clérambault, abbé de Saint-Taurin d'Évreux). La réflexion de d'Alembert entre en résonnance avec celles de Courbouzon (Éloge du marquis du Châtelet, I757) et de l'abbé Guillemin de Vaivre (Discours de réception, I774).

42 Éloge de Courbouzon par Grandfontaine.

43 Éloge de l'abbé d'Espiard.

44 Éloge du marquis de Montrichard.

45 Discours de réception.

46 Discours de réception. Le président de Vezet est installé à l'académie le 30 novembre I 778 , trois mois environ après Marnésia. 
ce soignée mais froide d'Isocrate la familiarité piquante, les saillies vives, inattendues, plaisantes du théâtre italien. Assujettis aux préjugés anciens, vous paraissez craindre de rassembler les genres qui ne semblent pas se rapprocher naturellement. Aussi, comme les savants, les orateurs, les littérateurs du siècle de Louis le Grand, vous n'osez pas, qu'il me soit permis de le dire, vous livrer à cette hardiesse de pensées qui embrasse et domine tous les sujets et subjugue presque tous les esprits. Fidèles au goût antique, vous adorez encore Homère, vous préférez Cicéron à Sénèque, Virgile à [lacune]. Rousseau, Racine, Molière, La Fontaine vous charment et vous ne blasphémez pas Boileau. Cette timidité, qui vous retient sur les traces des grands hommes qui vous ont précédés, vous ôte le mérite de frayer des routes nouvelles et ne vous permet pas d'occuper autant la renommée que ces auteurs qui, semblables aux coursiers indomptés, bondissent, s'égarent, s'abattent au milieu des précipices et, comme Icare, éternisent leurs écarts et leurs chutes.

On est décidément loin, dans ce passage, du ton ordinaire d'une réception. Interpeller les académiciens comtois réunis en séance publique ${ }^{47}$ pour émettre pareilles réserves quant à leurs talents, c'est piétiner avec impertinence les conventions, les convenances, les règles de courtoisie qui régissent un discours d'intronisation. Marnésia, au début du discours, déplorait pour lui-même « la facilité peu glorieuse de [se] traîner sur [les] traces » des prédécesseurs ; cette fois, ce sont ses confrères seuls qu'il critique pour leurs pratiques frileuses et sclérosantes; quant à lui, il réussit bel et bien à introduire l'inouï dans le cadre ritualisé de la réception. Bien que le procès-verbal de la séance n'en fasse pas état, on imagine la stupéfaction que le portrait a dû susciter chez les intéressés, peu habitués à entendre critiquer de la sorte leur goût et leurs pratiques classiques ${ }^{48}$. Marnésia, du reste, ne prolonge pas l'attaque ; son discours de réception s'achève sur des accents élogieux très normatifs. Remarquant que la « timidité » pourrait bien aussi procurer à l'académie quelques « avantages » - absence de polémique, maintien de la concorde, préservation de l'indépendance du jugement -, il salue les succès de ses confrères dans les champs de l'éloquence sacrée, judiciaire et historique, ainsi que dans les domaines des sciences et des beaux-arts, avant d'assurer que de ces réussites la mémoire durera toujours. L'immortalité académique, à défaut de l'éternité d'Icare...

Que Marnésia oublie le duc de Tallard en I777 n'empêche pas Droz de lui répondre chaleureusement et de l'inviter à résider souvent à Besançon, afin d'être titularisé. On ignore en revanche quelle fut la réponse de Nicolas-Gabriel Leclerc, médecin et vice-président de l'académie, au second

47 Ils sont au nombre de seize à cette séance solennelle du 24 août.

48 R. Bonnel a également relevé ce passage et la critique " acerbe " qui s'y déploie (op. cit., p. 95). 
discours de Marnésia, et si même il y en eut une : elle n'est en tout cas pas conservée dans les registres, et le procès-verbal de la séance n'en fait aucune mention. L'installation du marquis n'y donne d'ailleurs lieu qu'à ces lignes laconiques : « la séance a été terminée par l'annonce des sujets des prix et par un discours de M. de Marnésia sur les académies de provinces, à l'occasion duquel il a fait l'éloge de M. le marquis de Calviers ». Comme on le voit, c'est la conformité au rituel de la réception que met en avant le procès-verbal : seul l'éloge du prédécesseur est mentionné tandis que les écarts sont passés sous silence. Ainsi la portée transgressive du discours est-elle estompée.

Il serait tentant de voir dans la non-transcription de la réponse un indice de l'embarras des académiciens comtois à l'écoute de Marnésia, voire de leur répugnance à répondre à ses propos. Mais une telle interprétation serait téméraire, car d'autres discours de réception sont copiés sans réponse dans le fonds de l'académie, alors que leur contenu, beaucoup plus traditionnel que celui du marquis, n'est pas suceptible d'avoir déconcerté. Quoi qu'il en soit, on regrette que la réponse adressée à Marnésia soit introuvable. On aimerait savoir comment l'institution comtoise a réagi à ses propos, si conventionnels à certains égards et si déroutants par d'autres aspects, surtout pour elle qui défendait plutôt le conservatisme littéraire et politique. Lallusion laudative à d'Alembert, la réserve exprimée quant aux pratiques des Comtois étaient particulièrement de nature à heurter l'auditoire, plus habitué à entendre les récipiendaires fustiger la décadence du goût, la corruption de la philosophie ${ }^{49}$, les ravages du bel esprit et l'attrait pernicieux pour la nouveauté, perçue comme un ferment de désordre. Nombreux sont les impétrants qui, dans leur discours de réception, usent de la métaphore de la lumière aveuglante, de l'éclat éblouissant, pour dénoncer les écrits séduisants des écrivains et philosophes modernes ; si la figure d'Icare invoquée par Marnésia se rattache à cette image, le marquis ne rejette pas pour autant la nouveauté. Ne suggère-t-il pas contraire que le véritable académicien sait à la fois transmettre la tradition et la revivifier par quelques écarts? Aussi ses propos offrent-ils un intéressant contrepoint aux analyses de ses confrères. Pour achever d'en convaincre, on reproduira ici les fragments de deux autres discours de réception à l'académie de Besançon. Le premier est de François-Félix-Bernard Terrier de Santans (1734-I796), président à mortier au parlement de Franche-Comtéso ${ }^{\circ}$, pour qui

l'homme de lettres qui aspire à mériter des succès plus durables, loin de s'engager dans des routes inconnues, doit chercher la trace des grands hommes

49 Beaucoup s'évertuent à distinguer entre bonne et mauvaise philosophie.

50 Descendant d'une longue lignée de parlementaires comtois, il est, par sa mère, le neveu d'un autre académicien, Jean-François d'Espiard (I696-I778), abbé de Saint-Rigaud et prédicateur ordinaire de Marie Leszczynska. 
qui l'ont précédé, et jamais il ne doit marcher avec plus d'assurance que quand il les a pour modèles et pour guides ; il n'y a point de preuve plus sûre qu'on est égaré que quand on ne rencontre personne.

Nous sommes alors en I765, soit une douzaine d'années avant l'admission de Marnésia. Mais la même ligne est toujours défendue dans les années I780, et par le successeur même du marquis : l'abbé Jean-Joseh-Gabriel

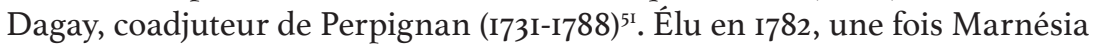
nommé honoraire, cet autre champion du conservatisme déplore à son tour les fourvoiements des gens de lettres et se réjouit de constater que la compagnie comtoise soit exempte de tels défauts :

\begin{abstract}
pour plaire, pour enlever les suffrages, pour se faire promptement un nom dans la littérature, il suffit souvent de s'écarter des routes ordinaires et d'enfanter avec assurance un système dont la nouveauté fait le mérite. L'espérance de réussir séduit aisément l'ardente imagination d'un jeune littérateur ; le désir de la célébrité le conduit insensiblement au mépris des principes les plus généralement reçus ; bientôt de vieilles vérités ne lui paraissent plus que de vieux préjugés, et le goût dominant du paradoxe finit par substituer des sophismes et des sarcasmes à l'autorité la plus respectable et la mieux établie. [...] Ce n'est pas ainsi que vous pensez, Messieurs...
\end{abstract}

Ainsi les titulaires successifs du même fauteuil s'expriment bien différemment à quelques années de distance. Les réserves et les audaces de Marnésia ne s'imposent guère à l'académie, d'autres récipiendaires encore le prouvent ${ }^{52}$. C'est pourquoi son discours de réception de 1778 est si captivant. Lisse de prime abord, mais laissant apparaître ici et là des aspérités inattendues, il bouscule un genre corseté et invite à imaginer ce que furent, au-delà du figement des versions officielles, les émotions, les réflexions, les réactions, non consignées par les archives, des académiciens comtois.

\title{
Bibliographie sélective
}

Bonnel, Roland, Éthique et esthétique du retour à la campagne au XVIII ${ }^{e}$ siècle, l'œuvre littéraire et utopique de Lezay-Marnésia, I735-I8oo, New-York, Bern, Peter Lang, 1995.

Manuscrits de l'académie des sciences, belles-lettres et arts de Besançon, conservés à la Bibliothèque municipale d'étude et de conservation de Besançon, accessibles sur le site Mémoire vive : patrimoine numérisé de Besançon (http://www.memoirevive.besancon.fr), onglet " Collec-

5I Son neveu et son frère sont également membres de l'académie de Besançon.

52 Par exemple l'abbé Nonnotte, reçu en I78I. 
tions » : « Institutions » : « Bibliothèques » : « Manuscrits de l'académie ». Voir en particulier les cotes : ms.academie.3 (discours de réception de Marnésia, I777) et ms.academie.8 (discours de réception de Marnésia, I778).

Roche, Daniel, Le siècle des Lumières en province : académies et académiciens provinciaux: $1680-1789$, Paris, EHESS, 1989 [1978].

Zoberman, Pierre, Les Cérémonies de la parole : léloquence d'apparat en France dans le dernier quart du XVII siècle, Paris, Champion, 1998.

Sur le marquis de Marnésia, on pourra également consulter :

Grappin, Pierre-Philippe, "Éloge des académiciens décédés, éloge de Lezay-Marnésia ", séance du 5 décembre I8I2, Académie des sciences, belleslettres et arts de Besançon, Besançon, Académie des sciences, belles-lettres et arts de Besançon, I812, pp. 73-78.

Tivier Henri, «Éloge du marquis de Lezay-Marnésia ", Mémoires de l'académie des sciences, belles-lettres et arts de Besançon, Besançon, Académie des sciences, belles-lettres et arts de Besançon, I879, p. I8. 